\title{
MODELACIÓN PRELIMINAR DEL FLUJO SUBTERRÁNEO EN UN SECTOR DE LA CUENCA INFERIOR DEL ARROYO DEL AZUL (BUENOS AIRES, ARGENTINA).
}

\section{PRELIMINARY GROUNDWATER MODELING OF A LOWER SECTOR OF ARROYO DEL AZUL BASIN (BUENOS AIRES, ARGENTINA)}

\author{
Erik Zimmermann $^{(1)(2)(*)}$, Luisa Fajardo ${ }^{(3)(4)}$ e Ilda Entraigas ${ }^{(3)(4)}$ \\ (1) Consejo Nacional de Investigaciones Científicas y Técnicas. Buenos Aires, Argentina. \\ ${ }^{(2)}$ Centro Universitario Rosario de Investigaciones Hidroambientales. Facultad de Ciencias Exactas, Ingeniería y \\ Agrimensura. Universidad Nacional de Rosario. Rosario, Santa Fe, Argentina.

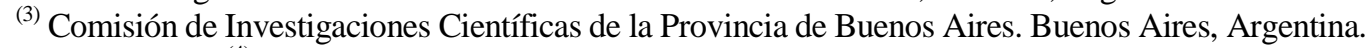 \\ ${ }^{(4)}$ Instituto de Hidrología de Llanuras. Azul, Buenos Aires, Argentina.
}

(*)e-mail: erikz@fceia.unr.edu.ar

\begin{abstract}
RESUMEN
Se construyó un modelo de flujo subterráneo, de carácter distribuido, con entradas y parámetros uniformes para todo el dominio de modelación. Este abordaje simplificado se realizó con el fin de caracterizar procesos generales y ajustar el modelo conceptual preliminar en el área de llanura del arroyo del Azul. Se propusieron metodologías a fin de completar las series discontinuas de niveles freáticos que fueron utilizados como condición de borde aguas anriba seleccionando una regresión lineal entre anomalías diarias de diferencias entre precipitación y evapotranspiración potencial con un retardo semanal con resultados de ajuste satisfactorios. Se consideraron como parámetros globales de calibración la transmisividad del acuífero, el coeficiente de almacenamiento, un coeficiente de recarga, una profundidad crítica para la evapotranspiración y un coeficiente de forma para la curva evapotranspiración vs. profundidad freática. Los coeficientes de eficiencia de NashSutcliffe entre niveles modelados y observados en 6 pozos de control oscilaron entre 0.58 y 0.72 . Tras la calibración, se concluyó que los parámetros obtenidos permiten una representación satisfactoria de la evolución de los niveles freáticos en los pozos de observación y de los patrones espaciales de las isofreáticas. Se pone en evidencia el rol prominente del proceso de evapotranspiración, el cual impacta directamente en el acuífero, representando una salida relevante del sistema. En el período de simulación de 43 meses, de características levemente secas, la recarga constituyó el 17 \% de la precipitación, el 87 \% de la misma retornó a la atmósfera como evapotranspiración, y el acuífero abastecería el 12.7 \% de la evapotranspiración potencial del período. Se considera que la modelación simplificada permitió una aceptable representación del comportamiento del acuífero freático.
\end{abstract}

Palabras clave: modelación flujo subterráneo, área de llanura, evaporación freática

\begin{abstract}
A distributed groundwater flow model has been constructed, with aggregated inputs and parameters for the entire modeling domain. This simplified approach was carried out in order to characterize general processes and adjust the preliminary conceptual model of the sector. Methodologies to complete the discontinuous series of water tables, which were used as the upstream condition, have been proposed. A linear regression between daily precipitation minus evapotranspiration anomalies with a weekly delay and water table levels was selected due to its good results. The aquifer transmissivity, the storage coefficient, a recharge coefficient, a critical depth for evapotranspiration and a shape coefficient for the evapotranspiration vs. water table depth curve were considered as global calibration parameters. The Nash-Sutcliffe efficiency coefficients between modeled and observed levels in six wells ranged between 0.58 and 0.72 . After the calibration, it can be affirmed that the parameters obtained allow a satisfactory representation of the evolution of observed groundwater levels and of the spatial patterns of the iso-phreatic curves. The prominent role of the evapotranspiration process is evident, which directly impacts in the aquifer, which representing a very relevant output from the system. In the simulation period of 43 months, with dry characteristics, the recharge constituted $17 \%$ of the precipitation, $87 \%$ of it returned to the atmosphere as evapotranspiration, and the aquifer supplied the $12.7 \%$ of the ETo of the period. It was considered that the simplified modeling allowed an acceptable representation of the water table dynamics.
\end{abstract}

Keywords: groundwater modeling, flatland area, phreatic evaporation. 


\section{INTRODUCCIÓN}

Los avances de la Hidrología Clásica se han desarrollado bajo el concepto de cuenca hidrográfica convencional, en el cual se define claramente un área de aporte, una organización de la red de drenaje y puede identificarse cada componente de la red con un orden de jerarquía.

El concepto clásico de cuenca, en sentido estricto, refleja sólo una parte de los posibles sistemas superficiales reales. La expansión del conocimiento a nivel mundial enfrenta a los hidrólogos con las grandes llanuras de muy baja pendiente en las cuales no se manifiesta una red de drenaje lineal o una superficie tributaria, manifestándose transferencias en sus divisorias, con pluralidad de puntos de salida en algunos casos, y dada la insuficiente pendiente las respuestas a los estímulos pluviales se dan en términos de acumulación. Son los localmente denominados Sistemas Hidrológicos No Típicos (SHN) (Caamaño Nelli et al., 1979).

La morfología de estos últimos sistemas condiciona los procesos hidrológicos y el modelo conceptual requerido para describirlos (Fertonani y Prendes 1983; Kovacs, 1983; Tricart, 1983; Caamaño Nelli y Zimmermann, 1990). Al respecto, la Hidrología Comparada (Falkenmark y Chapman, 1989) ofrece un encuadre metodológico para el análisis de los sistemas hidrológicos en general contemplando estas particularidades.

Hay un aspecto clave en el funcionamiento hidrológico de esos sistemas que es la marcada interacción de la hidrología superficial con la subterránea. En función de los niveles freáticos los excedentes hídricos pueden incorporarse al perfil del suelo o generar derrames y acumulación superficial de consideración.

Por otro lado, el enfoque ecohidrológico, como mirada interdisciplinaria que relaciona la hidrología con la ecología y los complejos procesos en el ciclo del agua, ofrece para estos sistemas un desafío particular debido a las múltiples interacciones hidrológicas entre sus componentes asociadas con las comunidades vegetales que los habitan.

El presente trabajo se plantea en el marco del análisis de la conectividad hidrológica del sector de estudio. En su forma más simple, la conectividad es una descripción del grado de relación o asociación dentro de un sistema, y corresponde a un conjunto estructurado de relaciones espaciales y/o temporales entre las diferentes entidades que lo conforman (Kool et al., 2013). En principio, se distinguen dos tipos de conectividad. La conectividad estructural hace referencia a la arquitectura y composición de un sistema (Noss y Cooperrider, 1994), esto es, al tamaño, forma, ubicación y arreglo espacial de los elementos que lo componen (Calabrese y Fagan, 2004), y también a las relaciones físicas entre esos elementos (Kadoya, 2009). A su vez, la conectividad funcional está directamente relacionada con el grado y dirección de los desplazamientos a través del paisaje (Kadoya, 2009) que describen los lazos entre los diferentes elementos que lo componen (Calabrese y Fagan, 2004).

Aplicando estos conceptos a la conectividad hidrológica, se la define entonces como la conexión que se establece entre los elementos de una cuenca, siendo la conectividad estructural aquella referida a los patrones espaciales que se establecen en el paisaje (es decir, la distribución de las unidades ambientales que influyen en los patrones de transferencia del agua y en las trayectorias de flujo); y la funcional como aquella concerniente a cómo esos patrones espaciales interactúan con los procesos de la cuenca para producir escorrentía, conexión entre flujos y, por lo tanto, la transferencia de agua en la cuenca (Fajardo y Entraigas, 2017).

Como una primera fase de investigación, se propone un modelo matemático de flujo subterráneo, de estructura no compleja, con el fin de cuantificar los principales procesos hidrológicos que se verifican en la zona de estudio propuesta. Los resultados derivados permitieron deducir elementos conceptuales del funcionamiento hidrológico subterráneo como "sostén" de los procesos que se dan en superficie. En una etapa futura se contemplarán las evoluciones de niveles freáticos y flujos con la atmósfera para la búsqueda de las conectividades con las comunidades vegetales de superficie.

\section{AREA DE ESTUDIO}

La cuenca del arroyo del Azul se ubica en la zona central de la provincia de Buenos Aires, entre los $58^{\circ}$ $51^{\prime}$ y $60^{\circ} 10^{\prime}$ de longitud oeste y $36^{\circ} 09^{\prime}$ y $37^{\circ} 19^{\prime}$ de latitud sur (Figura 1). Abarca una superficie de 6237 $\mathrm{km}^{2}$ y como formas destacables se reconocen un subambiente serrano hacia el sur de la cuenca con altitudes por sobre los $200 \mathrm{msnm}$ (pendiente media del terreno del $5 \%$ ), y un sub-ambiente de llanura hacia el norte, por debajo de los $130 \mathrm{msnm}$ (con pendientes que varían entre 0.5 y $0.8 \mathrm{~m} / \mathrm{km}$ ), con una zona de transición entre 
ambos, caracterizada por suaves ondulaciones.
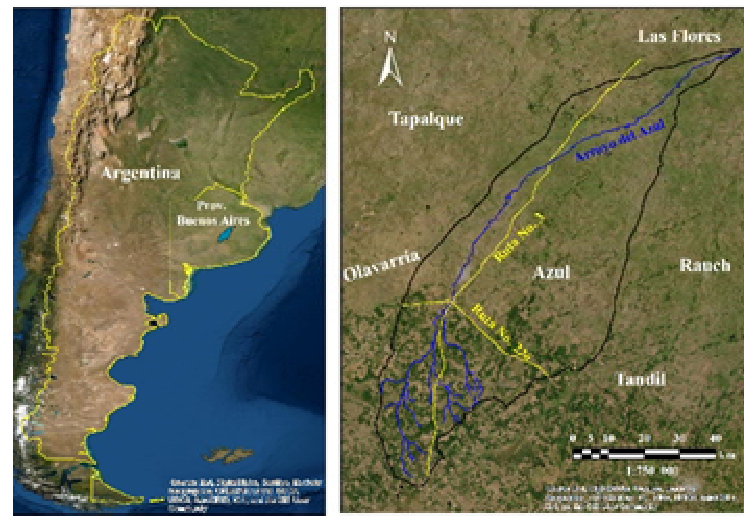

Figura 1. Ubicación relativa de la cuenca del arroyo del Azul, y principales localidades y vías de comunicación.

En el sector más bajo, sumamente llano, con sus suelos nátricos y drenaje deficiente, se desarrolla principalmente la cría ganadera extensiva en una matriz de pastizales naturales interrumpida por las numerosas cubetas y lagunas de carácter mayoritariamente semipermanente y por dunas parabólicas y longitudinales (Entraigas et al., 2019). Desde el punto de vista hidrológico, lo más notable del comportamiento del agua en cuencas tan deprimidas como la del Azul es la acumulación del agua sobre la superficie, y la interacción que se establece entre las aguas superficiales y las subterráneas, conformando un sistema hidrológico típico de las grandes llanuras. Específicamente se ha concentrado el interés en la modelación de un sector del área más deprimida de la cuenca (Figura 2) en el cual se ha realizado un seguimiento de coberturas vegetales, niveles freáticos, humedades del perfil y parámetros de suelo.

\section{Antecedentes geológicos y geomorfológicos}

Las Sierras de Azul están constituidas por afloramientos integrados por gneisses y rocas esquistosas precámbricas. En la cuenca del Azul, estudios de geoeléctrica señalan profundidades del basamento de hasta $120 \mathrm{~m}$ en la localidad de Azul y mayores a los $800 \mathrm{~m}$ en el área del paraje El Gualicho (desembocadura de la cuenca del Azul).

En lo que respecta a la composición de la cubierta sedimentaria del subsuelo dispuesta por encima del basamento, Méndez Escobar et al. (1995) consignan un acuñamiento de los depósitos del Mar Paranense (Mioceno verde) que se extiende hasta el norte de la ciudad de Azul, del Mioceno rojo hacia el sur de la localidad de Shaw (Figura 2), mientras que las arenas puelches se presentan un poco al norte de Shaw. En el sector superior de la cubierta consignan un espesor de depósitos que agrupan en la denominada sección epiparaniana, integrada por depósitos pampeanos $\mathrm{y}$ post pampeanos.

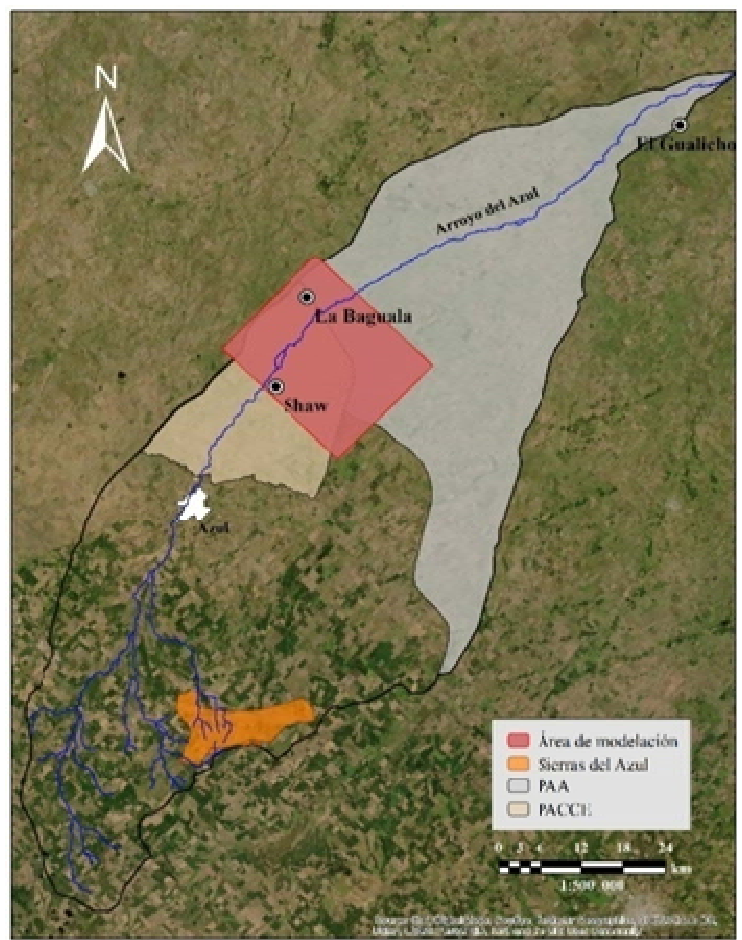

Figura 2. Zona de modelación y sitios referenciados.

Según Zárate y Mehl (2010), el sector bajo estudio se encuentra en el área extraserrana, comprendido entre las subunidades geomorfológicas denominadas planicie de agradación con costra calcárea estratiforme PACCE- y planicie de agradación aluvial -PAA(Figura 2). La PACCE está compuesta por limos castaño rojizos, compactos y carbonatados, con clastos de costras calcáreas, inmersos en una matriz limosa. Por encima de la costra calcárea se dispone una cubierta sedimentaria muy somera, de unos 0.50-0.70 $\mathrm{m}$ de potencia, muy modificada por pedogénesis. El sustrato suelo-costra calcárea genera encharcamientos superficiales, así como condiciones de drenaje interno deficiente en el perfil. El escaso gradiente generaría condiciones de escurrimiento muy lento. En cuanto a la PAA, está compuesta por una sección inferior de depósitos limos arenosos, castaño-rojizos, compactos, con estructuras de corriente y nódulos calcáreos. Por encima de ésta, se desarrolla una sección superior compuesta por limos finos gris cenicientos, con estratificación horizontal y eventual geometría lenticular correspondiente a facies palustres. La PAA 
funciona en la actualidad como un ambiente geomorfológico de abanico distal de muy bajo gradiente y escaso relieve relativo.

\section{Antecedentes hidrogeológicos}

El Instituto de Hidrología de Llanuras (IHLLA) dispone de dos redes de control piezométrico, una freática (6 m) y otra profunda (30 m), que cubren toda la cuenca del arroyo del Azul. La mayoría de los lugares de observación consisten en un par de piezómetros, uno de cada red. La superficie freática se encuentra cerca de la superficie del terreno (2-5 m) en casi toda la zona. La geometría de la superficie piezométrica a $30 \mathrm{~m}$ es similar a la freática; tanto es así que en el sector central hay varios emplazamientos donde los niveles piezométricos a 6 y a $30 \mathrm{~m}$ no son diferenciables en la práctica (Zabala et al., 2010).

El IHLLA (2003), en base a la interpretación de ensayos de bombeo y mediante una modelación matemática de acuíferos, presentó una estimación de parámetros hidráulicos para la cuenca del Azul (Tabla 1).

Tabla 1. Estimaciones de permeabilidades horizontales (Kh) y verticales (Kv) en la ciudad de Azul (Varni 2005).

\begin{tabular}{|c|c|c|c|}
\hline Profundidad(m) & Permeabilidad & $\mathrm{Kh}(\mathrm{m} / \mathrm{d})$ & $\mathrm{Kv}(\mathrm{m} / \mathrm{d})$ \\
\hline $0-40$ & Baja & 5 & 0.5 \\
\hline $40-60$ & Media & 15 & 8 \\
\hline $60-90$ & Baja & 10 & 5 \\
\hline $90-120$ & Alta & 30 & 20 \\
\hline
\end{tabular}

Varni (2005) infiere, a partir de la obtención de curvas de isoprofundidad mediante sondeos eléctricos verticales, que el basamento cristalino se encontraría entre los 200 m y 450 m en el sector de estudio.

Por otro lado, estima mediante balance de agua, balance de cloruros y modelación matemática de flujo subterráneo un entorno para la recarga freática entre el 8 y 15 \% de la precipitación.

El mismo autor ajusta mediante calibración valores de almacenamiento específico entre el 3\% y el 5\%.

\section{MODELO DE FLUJO SUBTERRÁNEO}

El modelo básico de flujo subterráneo consiste en la resolución numérica de las ecuaciones que gobiernan el movimiento no estacionario bidimensional (en planta) mediante una aproximación en diferencias finitas. La ecuación en derivadas parciales se expresa como:

$$
\frac{\partial}{\partial \mathrm{x}}\left(\frac{\left.\mathrm{T}_{\mathrm{x}} \partial \mathrm{h}\right)}{\partial \mathrm{x}}\right)+\frac{\partial}{\partial \mathrm{y}}\left(\frac{\mathrm{T}_{\mathrm{y}} \partial \mathrm{h}}{\partial \mathrm{y}}\right)=\mathrm{S} \frac{\partial \mathrm{h}}{\partial \mathrm{t}}+\mathrm{Q}
$$

donde: $\mathrm{T}_{\mathrm{x}}$ es la transmisividad del acuífero en la dirección x; $\mathrm{T}_{\mathrm{y}}$ es la transmisividad del acuífero en la dirección y; $\mathrm{S}$ es el coeficiente de almacenamiento del acuífero; h es la altura piezométrica; t es el tiempo; $\mathrm{x}, \mathrm{y}$ son coordenadas espaciales en planta y $\mathrm{Q}$ son funciones de fuente o sumidero expresadas como caudales netos por unidad de área.

A través de una aproximación en diferencias finitas, lo que implica reemplazar el continuo del sistema acuífero por un conjunto equivalente de elementos discretos (celdas), es posible obtener una solución numérica de la ecuación anterior.

En cada nodo o celda se plantea la ecuación de continuidad, considerando el almacenamiento de la celda y los flujos de intercambio con las vecinas y el exterior (Figura 3). Se considera el coeficiente de almacenamiento por celda, la ley de Darcy para el intercambio de flujos en el propio acuífero $\left(Q_{1}\right.$ a $\left.Q_{4}\right)$ y las recargas desde la zona no saturada $\left(Q_{5}\right)$ y las extracciones por bombeos y evapotranspiración $\left(\mathrm{Q}_{6}\right)$. Las ecuaciones planteadas en cada nodo deben resolverse para las principales incógnitas, que son los niveles $\mathrm{h}_{\mathrm{ij}}$. Para la resolución numérica de la ecuación diferencial se utiliza una forma modificada del método implícito iterativo de direcciones alternantes proporcionado por Prickett y Lonnquist (1968) a fin de resolver el conjunto de ecuaciones simultáneas. Esta técnica es incondicionalmente estable, independientemente del tamaño del incremento de tiempo.

Las recargas, por su lado, se consideran uniformes para el área de modelación, y se las define como un porcentaje de la precipitación mediante un coeficiente ajustable en el período de simulación $(\beta)$ :

$\mathrm{Q}_{5}=\beta \mathrm{P}$

Siendo $\mathrm{P}_{\text {y }} \mathrm{Q}_{5}$ la precipitación y la recarga diaria, respectivamente.

Para la evapotranspiración (ET) se consideró un coeficiente reductor $(\alpha)$ de la evapotranspiración potencial (ETo) como una función exponencial decreciente con la profundidad de la freática $(p f)$, desde la superficie hasta una profundidad 
denominada crítica $\left(p f_{c r i t}\right)$ :

$$
\begin{array}{ll}
\text { si pf }>\mathrm{pf}_{\text {crit }} & \alpha=0 \\
\text { si pf }<\mathrm{pf}_{\text {crit }} & \alpha=\exp (-\gamma \mathrm{pf}))
\end{array}
$$

Donde $\gamma$ es un parámetro que varía entre 0.4-1.0. Tanto $\gamma$ como $p f_{\text {crit }}$ se consideraron ajustables en las simulaciones y uniformes en el dominio.

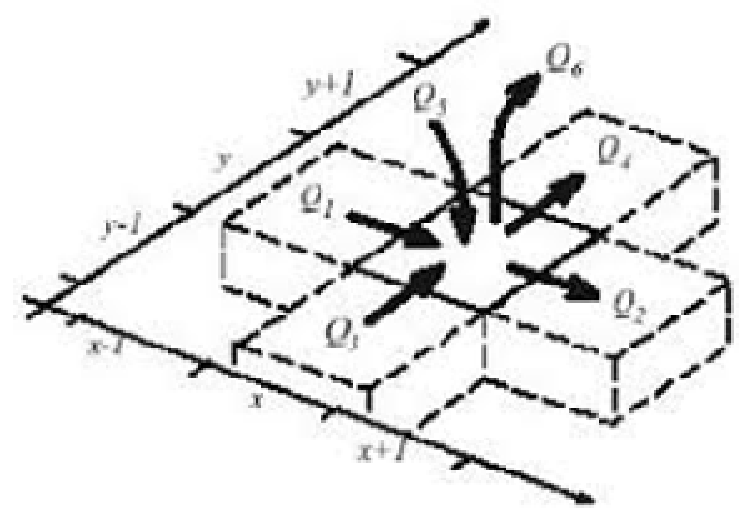

Figura 3. Discretización del dominio de modelación (extraída de Prickett y Lonnquist, 1968)

Para este trabajo se contó con el programa fuente original de Prickett y Lonnquist, escrito en FORTRAN IV, para acuíferos libres. El código, fue readaptado para adecuarlo a las condiciones de contorno del caso de estudio y las ecuaciones propuestas para la recarga y la evapotranspiración del acuífero.

\section{APLICACIÓN DEL MODELO}

\section{Grilla de modelación}

El dominio de modelación consistió en un rectángulo de $24 \mathrm{~km}$ en sentido SO-NE y $29 \mathrm{~km}$ en sentido NO$\mathrm{SE}$, el que se encuentra casi totalmente incluido en la cuenca superficial del arroyo del Azul (Figura 2). La grilla de discretización fue subdividida en elementos cuadrados de $1 \mathrm{~km}$ de lado, totalizando 696 celdas (24 filas, 29 columnas). El período de simulación del modelo se propuso en función de la información disponible, abarcando desde octubre de 2014 hasta abril de 2018 (43 meses), con un paso de cálculo temporal de 1 día.

\section{Condiciones de borde}

Las isofreáticas construidas a partir de los registros obtenidos en 21 pozos de observación ubicados en el área de estudio manifestaron un patrón de flujo repetitivo (Figura 4), cuya morfología permitió establecer hipótesis para las condiciones de borde. Este patrón reflejó un sentido predominante del flujo hacia el noreste, aproximadamente representativo en la totalidad del área de estudio, coincidente con la orientación de las márgenes laterales. Por ello las mismas fueron consideradas como líneas de flujo. El borde oeste del sector está próximo a la divisoria superficial de la cuenca del Azul, lo que justificaría la imposición de un borde impermeable. Para el límite este, las isopiezas del patrón de flujo sugieren la definición de una línea de flujo coincidente con dicho límite.

En los bordes aguas arriba (borde sur) y aguas abajo (borde norte) se propusieron condiciones de niveles y flujos preestablecidos, respectivamente (Figura 5). El borde sur cuenta con registros freatimétricos históricos en numerosos pozos de ese frente. Complementariamente se consideró la condición de control de flujos regionales hacia el río Salado en el borde norte.

Los registros históricos de niveles freáticos en el borde sur fueron discontinuos temporalmente $\mathrm{y}$ abarcaron pocas celdas representativas, por lo que fue necesario diseñar una estrategia de completamiento. Se contó con datos mensuales de seis pozos cuyos registros contemplaban dos períodos observados: 10/2014-03/2016 y 04/201704/2018.

En principio, se propuso completar los registros de niveles discontinuos mediante correlaciones con variables ambientales de patrones análogos, de las que, tras una selección previa, se consideraron las anomalías acumuladas de las diferencias entre precipitación y evapotranspiración (P-ETo) y de gravedad. Las anomalías acumuladas de P-ETo, denominadas anomalías hidrológicas (AH) se construyeron mediante la siguiente ecuación (Zimmermann, 2020):

$$
\mathrm{AH}_{\mathrm{i}}=\frac{\sum_{i=1}^{i} \mathrm{Pi}-\mathrm{ETo}_{\mathrm{i}}}{\frac{1}{N} \sum_{\mathrm{i}=1}^{N} \mathrm{Pi}-\mathrm{ETo}_{\mathrm{i}}}
$$

Donde: $i$ representa un día de cálculo, $N$, el número total de días del período, $P_{i}$, la precipitación del día $i$; $E T o_{i}$, la evapotranspiración potencial para el día $i$. 


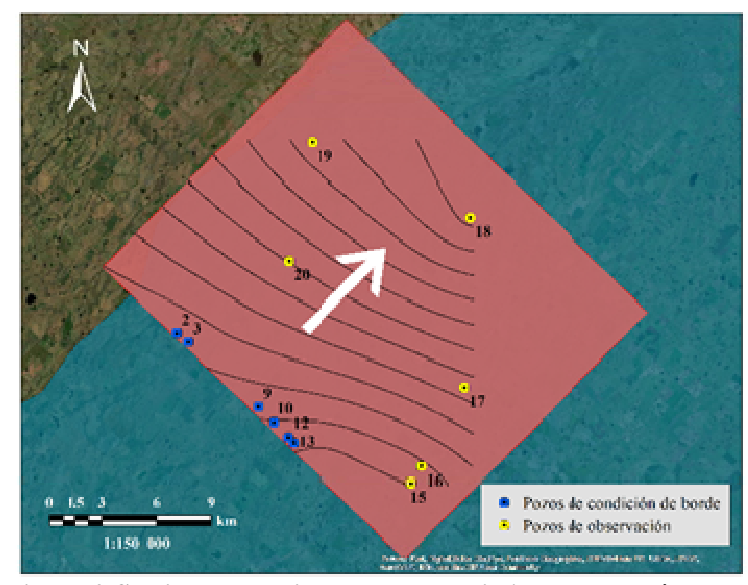

Figura 4. Sentido predominante del escurrimiento subterráneo en el sector modelado.
Las anomalías de gravedad, medidas desde una plataforma satelital, se refieren a cambios de masa en unidades equivalentes de agua líquida y fueron publicadas mensualmente en el marco del proyecto GRACE (Gravity Recovery and Climate Experiment).

En el sector de estudio las profundidades freáticas oscilan alrededor del metro, lo que pone de manifiesto que la evapotranspiración es una componente de suma importancia

Se contó con datos diarios de precipitación de dos sitios, la estación Azul Aero del Servicio Meteorológico Nacional (estación de confianza) y otra perteneciente a un establecimiento agropecuario cercano a la localidad de SHAW.

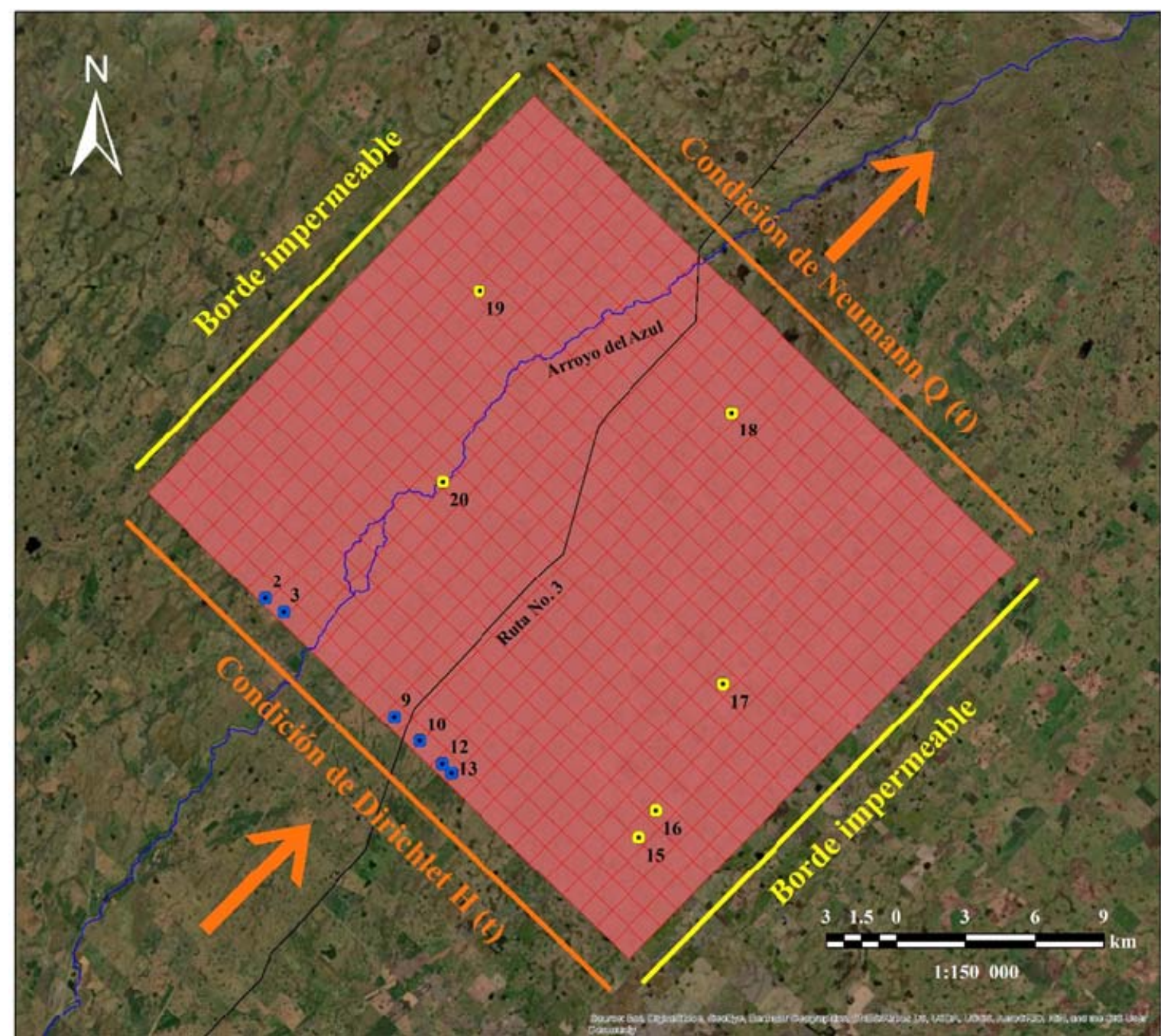

Figura 5. Grilla de modelación propuesta, condiciones de borde seleccionadas y pozos de observación de niveles freáticos utilizados para construir las condiciones del borde sur (amarillo) y para control del modelo (azul). 
Se dispuso de información de evapotranspiración potencial estimada mediante el método de PenmanMonteith y con un balance hidrológico calculado para el área de La Baguala (Varni y Custodio, 2013; Vercelli et al., 2019), ubicada en el interior del dominio de modelación (Figura 2).

Las anomalías hidrológicas, que fueron calculadas en forma diaria, se retrasaron entre un dia y una semana para contemplar un retardo entre el evento superficial y su efecto en los niveles freáticos. Se obtuvieron las mejores correlaciones con lag de 7 días.

Se optó por considerar las anomalías hidrológicas como metodología de completamiento temporal de registros freatimétricos por su mejor correlación (Tabla 2), definición temporal y disponibilidad de información (Zimmermann, 2020).

Los valores extrapolados temporalmente de los pozos permitieron completar el período de simulación en los pozos considerados.

Posteriormente, se extrapoló espacialmente esta información para la totalidad de celdas del borde mediante ecuaciones lineales. De esta manera pudo completarse la secuencia de datos freatimétricos en cada celda del borde sur, que fueron considerados como condición de contorno.

Tabla 2. Coeficientes de correlación entre anomalías y niveles freatimétricos de los pozos considerados.

\begin{tabular}{|c|c|c|}
\hline Pozos & $\begin{array}{c}\text { Anomalías } \\
\text { hidrológicas }\end{array}$ & $\begin{array}{c}\text { Anomalías } \\
\text { gravimétricas }\end{array}$ \\
\hline 2 & 0.893 & 0.794 \\
\hline 3 & 0.894 & 0.798 \\
\hline 9 & 0.861 & 0.624 \\
\hline 10 & 0.846 & 0.668 \\
\hline 12 & 0.881 & 0.748 \\
\hline 13 & 0.887 & 0.702 \\
\hline
\end{tabular}

Para las condiciones de borde norte, se consideró la condición de flujos regionales preestablecidos hacia el río Salado. El mismo se ubica a unos $150 \mathrm{~km}$ al $\mathrm{NE}$ en cuya dirección el gradiente regional topográfico es de aproximadamente $0.66 \mathrm{~m} / \mathrm{km}$ (valor estimado a partir de información altimétrica extraída de Google Earth ${ }^{\odot}$ ). Los caudales efluentes de cada celda se estimaron como producto entre el ancho $(1 \mathrm{~km})$, el espesor freático considerado, la permeabilidad horizontal de la celda y el gradiente regional estimado hacia el río Salado.

\section{Geometría estimada del acuífero freático}

Según registros estratigráficos, aproximadamente los primeros $40 \mathrm{~m}$ del acuífero freático están constituidos por sedimentos pampeanos de textura limosa cuyas permeabilidades se muestran en la Tabla 1.

Se consideró, de manera simplificada, un espesor de acuífero freático de $40 \mathrm{~m}$, consecuentemente con la hipótesis de flujo paralelo en ese espesor, de acuerdo con las observaciones piezométricas indiferenciadas entre el acuífero freático y aquellos más profundos (Zabala et al., 2010). Esto conlleva la suposición que la componente vertical hacia la superficie es despreciable lo cual, salvo en la descarga de la freática al arroyo del Azul que sería de carácter local, parece ser una hipótesis razonable.

\section{Condiciones iniciales}

Para fijar las condiciones iniciales de la simulación se consideraron los niveles de las celdas del borde sur en el mes de comienzo de la simulación, octubre de 2014. Se midieron los gradientes piezométricos en la dirección del flujo, considerando los niveles de los pozos interiores al dominio de modelado $(15,16,17$, 18, 19 y 20).

Se extrapolaron los niveles freáticos por celdas, teniendo en cuenta las cotas del borde sur y las pendientes mencionadas. Se revisaron las profundidades freáticas considerando cotas de superficie por celda (extraídas del modelo digital de terreno ajustado).

En casos donde el nivel freático superaba la cota del terreno, dichos valores fueron corregidos adjudicándoseles un valor mínimo equivalente a la profundidad radicular de la cobertura vegetal presente en esos sitios.

\section{CALIBRACIÓN DEL MODELO}

Teniendo presente las condiciones de borde, las recargas y descargas por celdas, conforme a la descripción previa del modelo, se realizaron las corridas pronosticando la evolución de niveles freáticos por celda en el período de simulación (octubre 2014abril 2018, totalizando 1308 días).

Se fijó un período de precalentamiento de 7 meses (octubre de 2014 - abril de 2015), para los cuales los resultados pueden estar influenciados por errores en las 
condiciones iniciales adoptadas. En consecuencia, el período con resultados confiables se extiende desde mayo de 2015 hasta abril de 2018 (3 años).

Se consideraron como parámetros de calibración la transmisividad del acuífero, $\mathrm{T}$; el coeficiente de almacenamiento, $S$; el coeficiente de recarga, $\beta$; la profundidad crítica, $p f_{\text {crit }}$ y el coeficiente de la curva de evapotranspiración vs. profundidad freática, $\gamma$.

Para comparar valores calculados por modelación y valores observados, se contó con los registros freáticos de los seis pozos en el dominio de modelación desde abril de 2017 hasta finalizar el período de simulación.

La Tabla 3 detalla los rangos considerados para los parámetros y los valores de ajuste alcanzados para minimizar las diferencias entre niveles estimados $\mathrm{y}$ observados. La metodología empleada para la calibración consistió en subdividir el rango de análisis de los parámetros en sub-intervalos y realizar simulaciones del modelo contemplando todas las combinaciones de parámetros posibles. Como función objetivo se consideró la eficiencia de Nash-Sutcliffe (NSE) entre los niveles simulados y observados en los
6 pozos internos del dominio para los períodos en los cuales se contaba con registros.

Tabla 3. Rango considerado de variación de parámetros y valores ajustados.

parámetros y valores ajustados.
\begin{tabular}{|l|c|c|}
\hline Parámetro & Rango evaluado & Valor calibrado \\
\hline$T\left(\mathrm{~m}^{2} / \mathrm{d}\right)$ & $100-2000$ & $\mathbf{2 0 0}$ \\
\hline$S(-)$ & $0.03-0.10$ & $\mathbf{0 . 0 3}$ \\
\hline$\beta(-)$ & $0.05-0.5$ & $\mathbf{0 . 1 7}$ \\
\hline$P f_{\text {crit }}(\mathrm{m})$ & $0.3-3.0$ & $\mathbf{3 . 3}$ \\
\hline$\gamma(-)$ & $0.4-1.0$ & $\mathbf{1 . 0}$ \\
\hline
\end{tabular}

\section{RESULTADOS ALCANZADOS}

Como se observa en la Tabla 3, los parámetros calibrados guardan mucha similitud con los hallados previamente por otros autores (IHLLA, 2003; Varni, 2005), lo que otorga una mayor certidumbre a los resultados.

La Figura 6 muestra la comparación de los registros freáticos calculados (líneas continuas) y observados (triángulos invertidos) para los parámetros calibrados en los 6 pozos de observación.

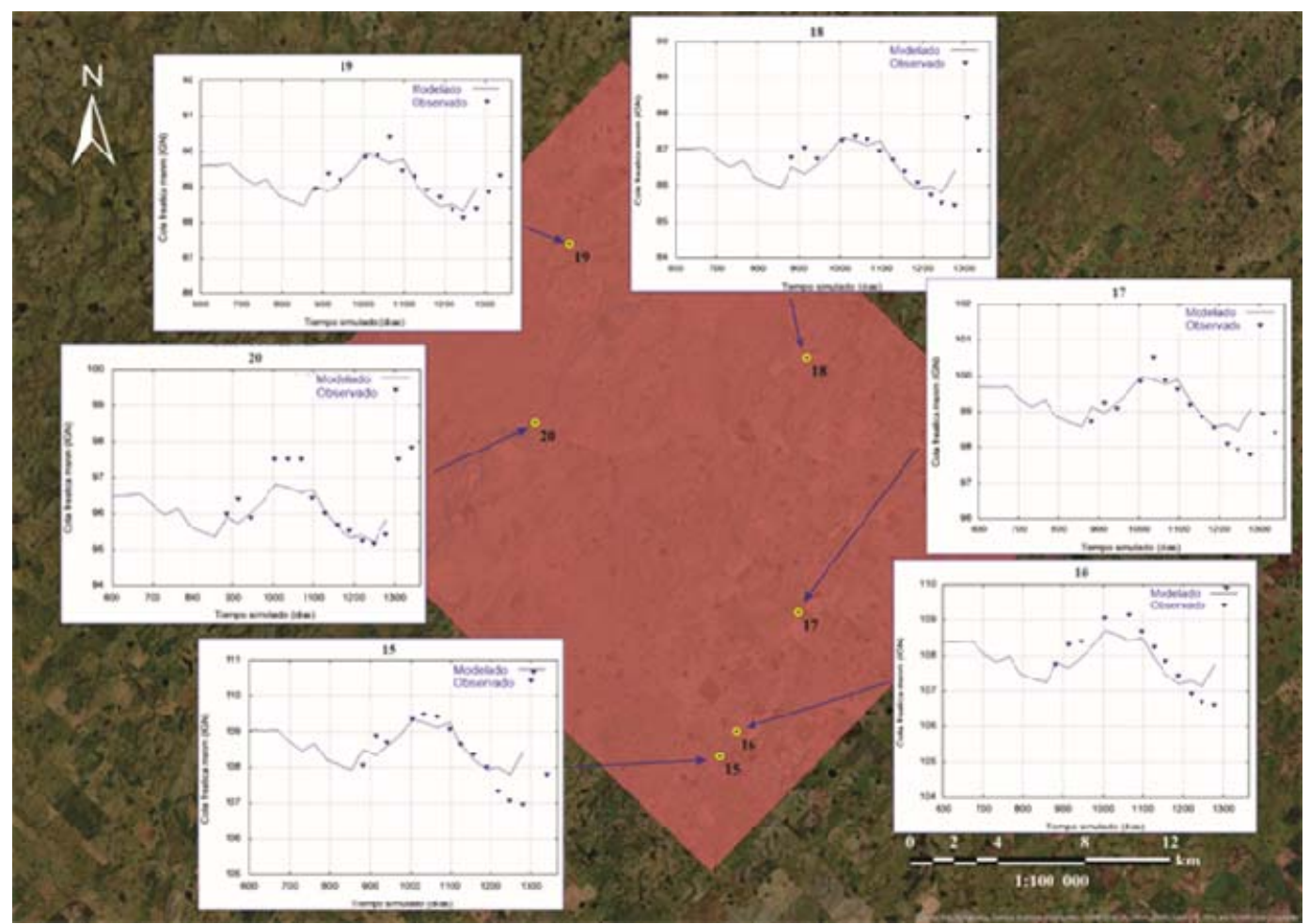

Figura 6. Evolución temporal de los niveles freáticos calculados por modelación (líneas continuas) y observados (triángulos invertidos) en los freatímetros seleccionados. 
La Tabla 4 muestra los coeficientes de eficiencia de Nash-Sutcliffe (NSE) para cada caso, donde se aprecia que el modelo refleja aceptablemente los niveles freáticos observados en la mayor parte de los pozos de control.

Tabla 4. Coeficientes de eficiencia de Nash-Sutcliffe (NSE) entre los niveles freáticos modelados y observados.

\begin{tabular}{|l|c|}
\hline $\begin{array}{c}\text { Pozos } \\
\text { de observación }\end{array}$ & NSE \\
\hline 15 & 0.5822 \\
\hline 16 & 0.6929 \\
\hline 17 & 0.6319 \\
\hline 18 & 0.6480 \\
\hline 19 & 0.7205 \\
\hline 20 & 0.6876 \\
\hline
\end{tabular}

Otro aspecto considerado fue el análisis del patrón espacial de las curvas isofreáticas, el cual resultó muy similar al observado en el prototipo (Figura 7), y se repite en otros mapas de isofreáticas calculados por el modelo.

El período de simulación descontado el precalentamiento abarcó un total de 1096 días (36 meses, comprendidos entre mayo de 2015 y abril 2018). De acuerdo con los guarismos del balance medio del área (Tabla 5), se deduce que el mismo coincide con un período de características levemente secas, y esto debe tenerse en cuenta en el análisis. En cuanto a la relación entre la ET y la recarga del acuífero, la misma es del $87 \%$, es decir que ese porcentaje de la recarga retorna a la atmósfera como evapotranspiración en el período analizado.

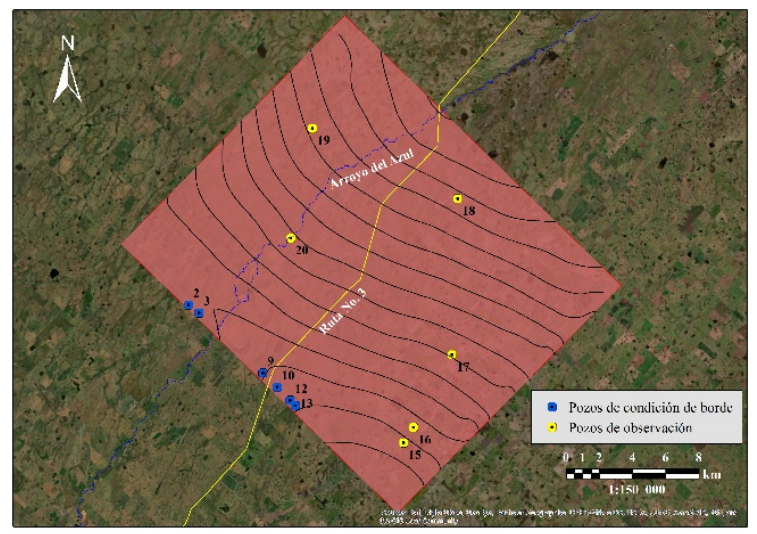

Figura 7. Curvas isofreáticas modeladas (abril de 2018).

Por último, a partir del análisis de la relación entre la evapotranspiración potencial y la estimada en la modelación, se desprende que el acuífero freático aporta el $12.7 \%$ de la ETo.
Tabla 5. Balance medio areal.

\begin{tabular}{|c|c|c|c|c|}
\hline & Precipitación & Recarga & ETo & $\begin{array}{c}\text { ET } \\
\text { modelada }\end{array}$ \\
\hline & $\mathrm{mm}$ & $\mathrm{mm}$ & $\mathrm{mm}$ & $\mathrm{mm}$ \\
\hline $\begin{array}{c}\text { Período } \\
2015 / 18\end{array}$ & 2648.7 & 450.3 & 3071.5 & 389.6 \\
\hline $\begin{array}{c}\text { Promedio } \\
\text { anual }\end{array}$ & $\mathbf{8 8 2 . 9}$ & $\mathbf{1 5 0 . 1}$ & $\mathbf{1 0 2 3 . 8}$ & $\mathbf{1 2 9 . 9}$ \\
\hline
\end{tabular}

Con la información obtenida de la evolución de los niveles freáticos para cada celda de la modelación, se proyecta analizar sus estadísticos, permanencias y frecuencias a lo largo del periodo de simulación. Se cree que esta información, deducida de los resultados del modelo, permitirá inferir conexiones entre la freatimetría y las coberturas vegetales de superficie para un amplio sector de la Pampa Deprimida.

\section{CONCLUSIONES}

Se construyó un modelo de flujo subterráneo, de carácter distribuido, con entradas y parámetros uniformes para todo el dominio de modelación. Este abordaje simplificado se realizó con el fin de caracterizar procesos generales y ajustar el modelo conceptual preliminar del sector.

Tras una calibración puede afirmarse que los parámetros obtenidos permiten una representación satisfactoria de la evolución de los niveles freáticos en los pozos de observación, como así también de los patrones espaciales de las isofreáticas.

Se pone en evidencia el rol prominente del proceso de evapotranspiración, el cual impacta directamente en el acuífero, representando una salida significativa del sistema.

Estos argumentos permiten considerar que la modelación simplificada permitió una aceptable representación del acuífero freático.

\section{REFERENCIAS}

Caamaño Nelli, G. E. y Zimmermann, E. (1990). Tipología de los Sistemas Hidrológicos Superficiales. XVI Reunión Científica de la Asociación Argentina de Geofísicos y Geodestas. Bahía Blanca. Argentina. Caamaño Nelli, G. E., Fertonani, M. E., Prendes, H. H. y Quinodoz, H. A. (1979) Analysis of Non-Typical Hydrological Systems. Theorical Bases and Methodology Criteria. Departamento de Hidrología 
General y Aplicada. Facultad de Ingeniería en Ciencias Hídricas. UNL. Santa Fe.

Calabrese, J. M. y Fagan, W. F. (2004). A comparisonshopper's guide to connectivity metrics. Frontiers Ecology and the Environment, 2(10):529-536.

Entraigas, I., Vercelli, N. y Fajardo, L. (2019). Plant communities along preferential superficial water flow paths across a floodplain landscape. Ecohydrology, 12(6): e2124. https://doi.org/10.1002/eco.2124

Fajardo, L. y Entraigas, I. (2017). Conectividad hidrológica en un sistema hidrológico no típico. Caso del sector llano de la cuenca del Arroyo del Azul. Informe de avance beca doctoral CIC.

Falkenmark, M., y Chapman, T. (1989). Comparati-ve hydrology: An ecological approach to land and water resources. Unesco.

Fertonani, M., y Prendes, H. (1983) Hidrología en áreas de llanura. Aspectos Conceptuales Teóricos y Metodológicos. Hidrología de Grandes Llanuras. Olavarría.Argentina. 1, p 119-156.

IHLLA. (2003). Sistema de soporte para la gestión eficiente de los recursos hídricos en la llanura bonaerense. Inédito, p 267.

Kadoya, T. (2009). Assessing functional con-nectivity using empirical data. Population Ecology, 51(1): 515.

Kool, J. T., Moilanen, A. y Treml, E. A. (2013). Population connectivity: recent advances and new perspectives. Landscape Ecology, 28(2): 165-185.

Kovacs, G. (1983). General Principles of Flat-lands hydrology. Hidrología de Grandes Llanuras. Olavarría. Argentina, 297-357.

Méndez Escobar, R., Usunoff, E. y Weinzettel, P. A. (1995). Aportes al conocimiento del subsuelo de la cuenca del arroyo Azul (provincia de Buenos Aires). V Jornadas Pampeanas de Ciencias Naturales. Santa Rosa, La Pampa. Tomo II, 55-60

Noss, R. F., y Cooperrider, A. Y. (1994). Saving nature's Legacy: Protecting and restoring biodiversity. Island Press, Washington, DC, p 416.

Prickett, T. A., y Lonnquist, G. G. (1968). Aquifer simulation program listing using alternating direction implicit method. Illinois State Water Survey mimeographed report presented at International Association of Scientific Hydrology Symposium on Use of Computers in Hydrology, Tucson, Arizona.

Tricart, J. (1983) L'Hydrologie des Grans Plains, Quelques Reflexions Methodologiques. Hidrología de las grandes Llanuras. Olavarría, Argentina, 2, p 1191-1193.

Varni, M. (2005). Evaluación de la recarga al acuífero del Azul: Valores medios y variaciones temporal y espacial. (Tesis doctoral). Facultad de Ciencias Exactas, Ingeniería y Agrimensura. UNR.

Varni, M., y Custodio, E. (2013). Evaluación de la recarga al acuífero del Azul: 1. Análisis de las características climáticas. En VIII Congreso Hidrogeológico Argentino y VI Seminario Hispano Latinoamericano sobre temas actuales de hidrología subterránea, La Plata. Argentina. doi: 10.13140/2.1.4000.2560.

Vercelli, N., Varni, M., Lara, B., Entraigas, I. y Ares, G. (2019). Linking soil water balance with flood spatial arrangement in an extremely flat landscape. Hydrological Processes, 34 (1): 21-32. doi: 10.1002/hyp.13567.

Zabala, M. E., Manzano, M., y Vives, L. (2010). Estudio preliminar del origen del fondo químico natural de las aguas subterráneas en la cuenca del arroyo del Azul. Hacia la gestión integral de los recursos hídricos en zonas de llanura. Volume IM, Varni, I. Entraigas y L. Vives (Eds), 249-256.Azul, Buenos Aires, Argentina.

Zárate, M., y Mehl, A. (2010). Geología y geomorfología de la cuenca del arroyo del Azul, provincia de Buenos Aires. Argentina. Hacia la gestión integral de los recursos hídricos en zonas de llanura, 1, 65-78.

Zimmermann, E. (2020) Completamiento de series temporales de niveles freáticos en un área deprimida de la llanura bonaerense. Cuadernos del CURIHAM. 26:55-61. doi: 10.35305/curiham.v26i0.148

\section{Tipo de Publicación: ARTICULO.}

\section{Trabajo recibido el 03/10/2020 y aprobado para su publicación el 18/11/2020.}

\section{COMO CITAR}

Zimmermann, E.; Fajardo, L. y Entraigas, I. (2020). Modelación preliminar del flujo subterráneo en un sector de la cuenca inferior del arroyo del Azul (Buenos Aires, Argentina). Cuadernos del CURIHAM. 26: 21-30. DOI: https://doi.org/10.35305/curiham.v26i0.154

Este es un artículo de acceso abierto bajo licencia: Creative Commons Atribución - No Comercial Compartir Igual 4.0 Internacional (CC BY-NC-SA 4.0) (https://creativecommons.org/licenses/by-ncsa/4.0/deed.es) 